\title{
Balkanologie
}

Balkanologie Revue d'études pluridisciplinaires

Vol. V, no 1-2 | 2001

Volume V Numéro 1-2

\section{L'économie du nettoyage ethnique à Prnjavor}

\section{Nikola Guljevatej}

\section{OpenEdition}

\section{Journals}

Édition électronique

URL : http://journals.openedition.org/balkanologie/693

DOI : $10.4000 /$ balkanologie.693

ISSN : 1965-0582

\section{Éditeur}

Association française d'études sur les Balkans (Afebalk)

Édition imprimée

Date de publication : 31 décembre 2001

ISSN : 1279-7952

\section{Référence électronique}

Nikola Guljevatej, «L'économie du nettoyage ethnique à Prnjavor», Balkanologie [En ligne], Vol. V, n 1-2 | 2001, mis en ligne le 02 juin 2008, consulté le 17 décembre 2020. URL : http://

journals.openedition.org/balkanologie/693; DOI : https://doi.org/10.4000/balkanologie.693

Ce document a été généré automatiquement le 17 décembre 2020.

(c) Tous droits réservés 


\title{
L'économie du nettoyage ethnique à Prnjavor
}

\author{
Nikola Guljevatej
}

1 La commune de Prnjavor se situe dans le nord de la Bosnie-Herzégovine, où elle occupe $631 \mathrm{~km}^{2}$. En 1991, elle comptait 47055 habitants, dont 71,2\% de Serbes, 15,2\% de Musulmans / Bochniaques, 3,7 \% de Croates et autant de Yougoslaves. De plus, 6,2 \% de la population rentrait dans la catégorie "Autres", celle-ci incluant, par ordre d'importance décroissante, des Ukrainiens, des Italiens, des Tchèques et des représentants de douze autres minorités non-constitutives ${ }^{1}$. En 1999, l'International Police Task Force (IPTF) a estimé que la population totale de Prnjavor était passée de 47055 à 53000 personnes environ, du fait notamment de l'afflux de déplacés serbes. La composition ethnique de la commune serait la suivante : Serbes $85 \%$, "Autres" $13 \%$, Bochniaques $1,5 \%$ et Croates $0,5 \%{ }^{2}$. L'homogénéisation sur des critères ethniques a donc bien eu lieu, comme dans toute la Bosnie-Herzégovine.

2 Une des thèses souvent avancées pour expliquer le nettoyage ethnique évoque des "haines implacables de l'autre". Cette idée de "haines séculaires" implique que toutes les minorités auraient dû connaître le même sort tragique. Or, le cas spécifique de Prnjavor remet en cause une telle explication. Contrairement à d'autres villes de taille comparable qui ont fait la une des médias internationaux (Srebrenica, Prijedor, etc.), Prnjavor est restée dans l'ombre. Il n'y eut pas d'atrocités à grande échelle, ni d'expulsion massive et organisée, ni d'exécutions sommaires, ni de camps de concentration. Il apparaît aussi que le nettoyage ethnique a touché beaucoup plus les Bochniaques et les Croates que les minorités non-constitutives, qui constituent pourtant une part non-négligeable de la population locale.

3 Le cas de Prnjavor soulève donc plusieurs questions. Pourquoi une telle "modération" dans les formes du nettoyage ethnique? Pourquoi une telle "clémence" envers les minorités non-constitutives? Le retour s'en trouve-t-il pour autant facilité? 


\section{Les populations de la commune de Prnjavor}

4 Il est des localités dont le nom même renvoie à leur insignifiance. Prnjavor est l'un de ces lieux-là. Le mot "prnjavor" désigne dans la langue courante un lieu éloigné des chemins principaux et difficile d'accès, mais aussi, plus spécifiquement, un village construit sur le domaine d'un monastère ${ }^{3}$. Dès lors, faut-il être surpris de rencontrer souvent ce toponyme en Bosnie, dans les Balkans? Un magazine de Banja Luka intitulait son reportage sur Prnjavor, à $50 \mathrm{~km}$ de là : «La ville dont on ne se souvient pas $»^{4}$. Cependant, Prnjavor a une particularité dont ses habitants étaient, et sont encore parfois, particulièrement fiers : cette commune comptait en 1991 dix-huit nationalités différentes, et était la championne incontestable de la pluriethnicité en Bosnie-Herzégovine.

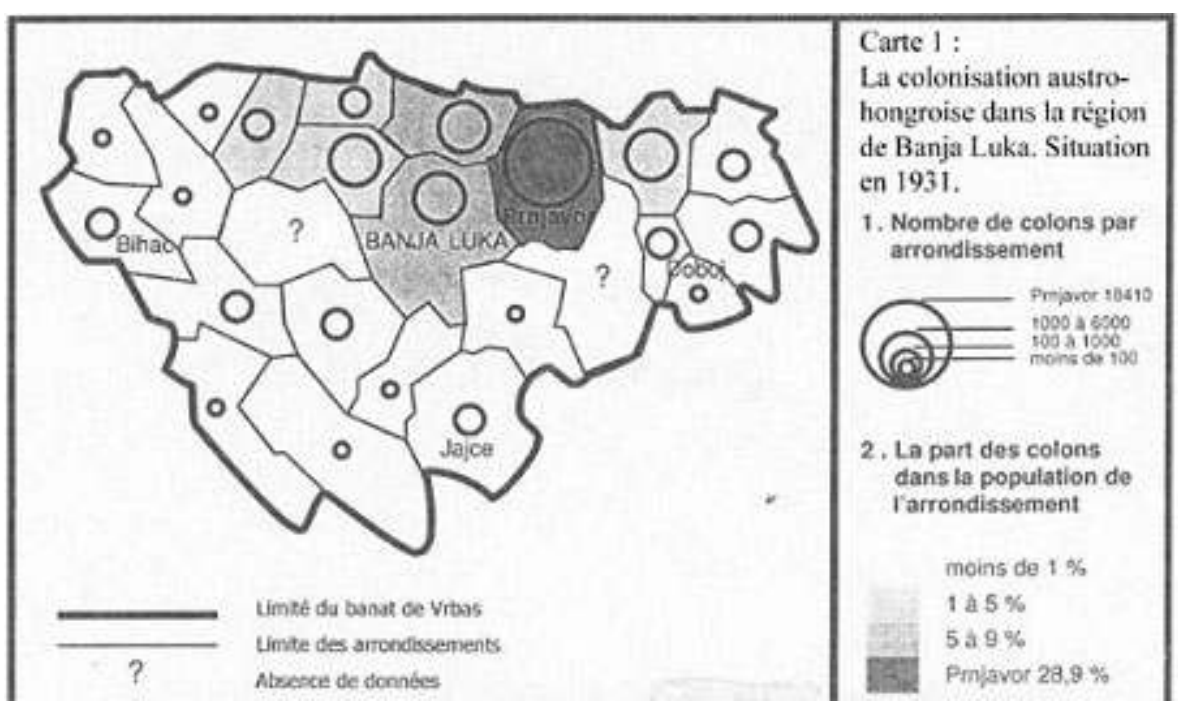

On ne peut avoir de certitudes sur les origines de la population de Prnjavor. On sait seulement que la majorité catholique, qui peuplait la région au Moyen Âge, a disparu pendant la période ottomane (entre 1530 et 1878) pour céder la place aux populations orthodoxe et musulmane. Le premier recensement austro-hongrois de 1879 dénombrait dans l'arrondissement de Prnjavor 17009 orthodoxes (85,3\%), 2700 musulmans $(13,5 \%)$ et 243 catholiques $(1,2 \%)$. En cela, Prnjavor est représentatif de la Bosnie dans son ensemble, et de la région de Bosanska Krajina en particulier.

Tableau 1 : L'évolution confessionnelle de l'arrondissement de Prnjavor (1879-1931)

\begin{tabular}{|r|r|r|r|r|r|}
\hline & Orthodoxes & Musulmans & Catholiques & Juifs et autres & Total \\
\hline 1879 & 17009 & 2700 & 243 & - & 19952 \\
\hline 1885 & 20226 & 3186 & 1001 & 11 & 24406 \\
\hline 1895 & 24334 & 4769 & 1199 & 95 & 30424 \\
\hline 1910 & 28202 & 3578 & 13732 & 802 & 46314 \\
\hline 1921 & 28604 & 3827 & 16590 & 872 & 49893 \\
\hline
\end{tabular}




\begin{tabular}{|c|c|c|c|c|c|}
\hline 1931 & 39964 & 4669 & 19061 & 929 & 63653 \\
\hline
\end{tabular}

6 La palette des nationalités s'élargit au tournant du siècle, avec un afflux massif de colons venus de tous les coins de l'Empire austro-hongrois pour trouver des terres à défricher ${ }^{5}$. Ils y étaient incités par des exemptions fiscales destinées à améliorer l'agriculture locale, avec en dernière instance la perspective pour Vienne d'augmenter les revenus du gouvernement provincial ${ }^{6}$. D'autres estiment que "l'Autriche-Hongrie voulait à tout prix diminuer la grande concentration de Serbes dans cette région $»^{7}$. Quoi qu'il en soit, cette entreprise de colonisation fut limitée au nord de la Bosnie (voir Carte 1). Alors que dans le reste de la Bosnie-Herzégovine, les Austro-Hongrois arrivaient au compte-goutte pour occuper des postes de cadres industriels et administratifs, Prnjavor connut surtout une immigration paysane. L'arrondissement eut par conséquent la plus forte croissance démographique de toute la BosnieHerzégovine entre 1885 et $1910:+52,2 \%$. La croissance externe (colonisation) atteignit au total $+27 \%$ entre 1895 et 1910, soit en moyenne $+1,8 \%$ par an, si bien qu'en 1931, le serbo-croate n'était pas la langue maternelle de plus de 18000 personnes, soit $29 \%$ de la population totale de l'arrondissement, un record en Bosnie-Herzégovine.

7 Les premiers à s'installer, peu après l'entrée des troupes austro-hongroises en 1878, furent des Allemands de Silésie et de Rhénanie, sur l'invitation expresse du premier archevêque de Bosnie-Herzégovine, Mgr. Stadler. Leur installation se fit par colonies agricoles : deux groupes de 20 et 70 familles allemandes respectivement obtinrent les meilleures terres au nord et à l'est de la commune, à Šibovska et Glogovac, où l'on peut aujourd'hui encore reconnaître un parcellaire égalitaire autour de ces nouveaux villages, à la forme caractéristique de villages-rues. Les Allemands apportèrent des techniques nouvelles d'exploitation agricole et développèrent les premières activités industrielles (moulins à vapeur). Après eux vinrent les Hongrois, 40 familles environ, concentrées dans le village préexistant de Lužani. Leur histoire se termina pendant la Seconde Guerre mondiale, en 1942, lorsque la Wehrmacht organisa l'évacuation des Allemands et des Hongrois qui le souhaitaient. Les rares qui restèrent jusqu'en 1945 ne connurent donc pas l'expulsion massive qui frappa surtout les Allemands de Voïvodine, en même temps que ceux de toute l'Europe de l'Est. En 1948, il ne restait plus que six Allemands et quatre Hongrois dans la commune de Prnjavor.

8 Parmi les colons dont il ne reste plus de traces significatives, il faut aussi mentionner les Polonais. Ils arrivèrent de la région de Cracovie dans les années 1890, et furent dispersés sur l'ensemble de la commune par une sorte de tirage au sort des parcelles que les pouvoirs locaux leur avaient attribuées. Après la Seconde Guerre mondiale, la Pologne invita tous les Polonais de l'extérieur à rentrer au pays, et les autorités yougoslaves organisèrent leur rapatriement entre novembre 1945 et avril 1946. Ceux de Prnjavor firent partie des 20000 Polonais rapatriés, si bien que, en 1948, il en restait moins d'une centaine.

9 Les trois principales communautés de colons qui survécurent à la Seconde Guerre mondiale furent les Italiens, les Tchèques et les Ukrainiens.

10 Selon les dires des anciens, les Italiens sont arrivés en 1881 de la région de Valsugano, dans le Tyrol, poussés par les inondations du Brento et la faillite de la culture de la soie dans cette région de l'Empire austro-hongrois. Ils étaient au début installés dans quatre 
villages différents mais, après maintes lettres de doléance aux autorités, ils obtinrent leur regroupement dans le village de Štivor.

11 Les Tchèques installés à Prnjavor ont vécu une véritable odyssée : il s'agissait de paysans tchèques partis en Russie en 1874 à l'invitation du Tsar, pour en revenir vingt ans plus tard lorsqu'on exigea d'eux qu'ils deviennent citoyens russes, ce qu'ils refusèrent. L'Autriche-Hongrie leur accorda des terres en Croatie, dans les environs de Daruvar, et en Bosnie-Herzégovine, à Nova Ves et à Prnjavor, où 25 familles fondèrent le village de Maćino Brdo.

Les Ukrainiens de Galicie autrichienne arrivèrent les derniers. Les fortes densités de population rurale et le retard de l'industrialisation ont destiné cette région à fournir un grand nombre d'immigrants aux Etats-Unis et au Canada, mais aussi quelque 8000 colons à l'arrondissement de Prnjavor entre 1898 et 1913. Ce n'est qu'en 1901 que les autorités austro-hongroises, freinées par l'hostilité du Parlement provincial de BosnieHerzégovine, leur distribuèrent des terres dispersées entre plusieurs villages de l'arrondissement. Confrontés à un risque d'assimilation ${ }^{9}$, ils se dotèrent de plusieurs paroisses et de diverses associations culturelles entre 1900 et 1917.

\section{Formes d'identification au territoire}

13 La répartition des différentes communautés sur le territoire de Prnjavor montre une mixité élevée en ville et une forte séparation dans les campagnes, si bien que l'on peut parler de connotation identitaire des lieux de résidence. Dans la campagne, qui englobe $82,2 \%$ de la population locale, cette identification communautaire se fait à l'échelle des hameaux et des villages. Dans la ville de Prnjavor, elle se fait à l'échelle des quartiers (mahala).

\section{a) Faible mixité des campagnes}

Sur 62 villages, 55 présentent les caractéristiques de villages dispersés, et comptent plusieurs hameaux (voir Carte 2). Tous ces villages dispersés sont habités par de très fortes majorités serbes, ce qui est le signe d'une création relativement récente, contemporaine de l'arrivée des Serbes dans la région. Ils se distinguent des autres villages par leurs hameaux, qui portent fréquemment le nom des familles qui les habitent (par exemple : Vasići, Božunovići et Milići à Grabik Ilova). D’autres toponymes de lieux les entourant comprennent aussi des indicatifs d'appartenance à une famille ou un individu (par exemple : Radiškovića Brdo -la colline des Radišković- au sud-est de Prnjavor). Ces toponymes liés aux noms de famille indiquent leur relative jeunesse. Ce fait est particulièrement flagrant autour de la Careva Gora, la "forêt du Tsar", défrichée par les nouveaux venus. 


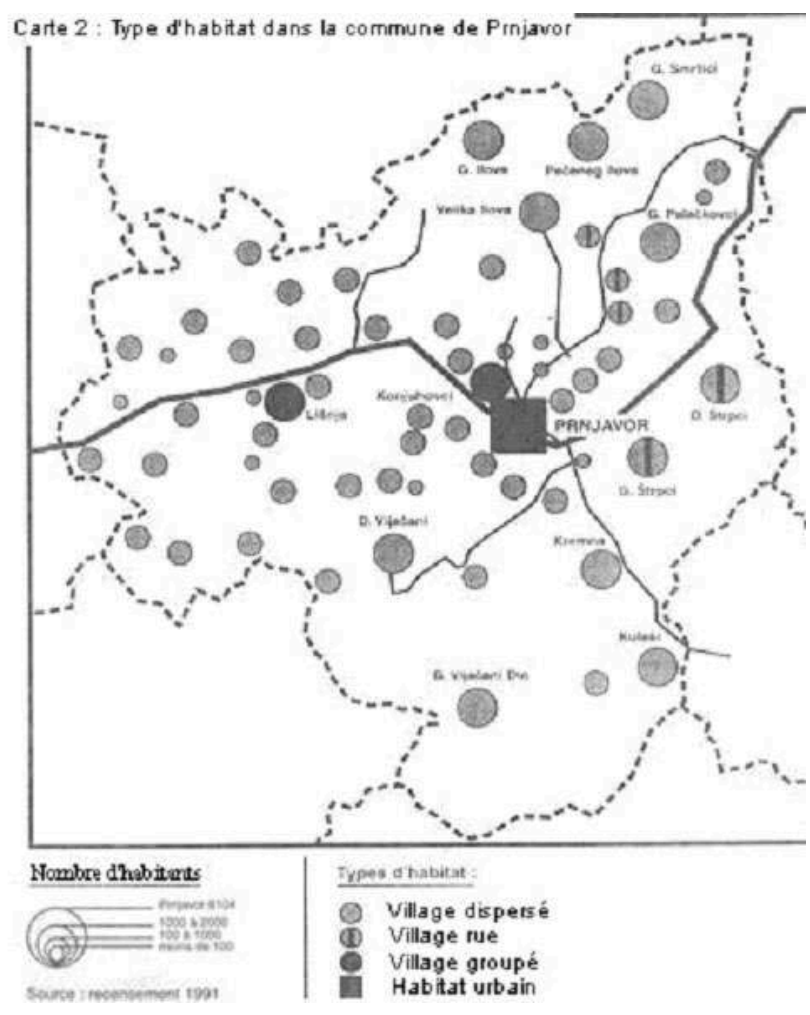

15 Ces toponymes indiquent également la stabilité des structures familiales patriarcales au sein de la zadruga ${ }^{10}$. Avant que la société ne se modernise partiellement, l'appropriation d'un terrain était inséparable de l'appartenance familiale : un homme dénommé Vaso s'installait avec sa famille à l'écart des routes en défrichant un bout de forêt; ses fils poursuivaient le défrichage et s'installaient avec leurs familles à côté de la maison du père : le hameau portait alors le nom de "Vasići". Aujourd'hui encore, tous les habitants d'un hameau sont donc des cousins plus ou moins proches, se reconnaissant comme tels. Le nom de famille est dès lors porteur d'une identité territoriale, mais aussi religieuse et nationale, vu que l'exogamie est très faible dans ce type de village.

Ce type d'identification au hameau familial ne se retrouve pas chez les Bochniaques. Leur identification au territoire se fait principalement à l'échelle du village : en 1991, les Bochniaques urbains forment 29 \% de la population du chef-lieu Prnjavor. Par ailleurs, 82,7 \% des Bochniaques ruraux vivent dans cinq villages où ils forment la majorité de la population (entre 60 et $99 \%$ des habitants) ; les autres sont répartis entre 19 villages où ils constituent une forte minorité.

On remarque que les villages où les Bochniaques sont majoritaires se trouvent le long des axes de communication locaux proches des meilleures terres agricoles, réservées aux musulmans à l'époque ottomane. Inversement, la prédilection des Serbes de Prnjavor pour les endroits les plus reculés vient d'une série de lois très contraignantes pour les chrétiens à l'époque ottomane, les Serbes représentant à l'époque la majorité des kmets (serfs), du moins là où l'on a pu l'établir avec certitude. C'est ce qui explique leur dispersion à la périphérie de la commune, sur les anciennes "terres désertes" (erazi mevat) que la loi de Ramazan de 1858 définissait comme celles se trouvant à une demiheure de marche de tout lieu habité, ou hors de portée de la voix humaine, soit un kilomètre et demi ${ }^{11}$. 
que l'on vient de ces villages-là indique très souvent de façon extrêmement précise son identité religieuse et nationale. Cette règle valait aussi, et vaut encore parfois, pour les communautés de colons qui ont été installées sur des terres libres, et non dans des villages préexistants : les Italiens étaient regroupés dans leur village de Štivor, les Tchèques à Maćino Brdo, et les Allemands à Glogovac et à Šibovska (voir Carte 3). Ces derniers ont gardé leur forme de village-rue (voir Carte 2), même s'il n'y a plus d'Allemands aujourd'hui. Mais cette règle ne vaut pas pour les colons arrivés plus tardivement, les Ukrainiens et les Polonais, du fait de leur dispersion sur tout le territoire de la commune.

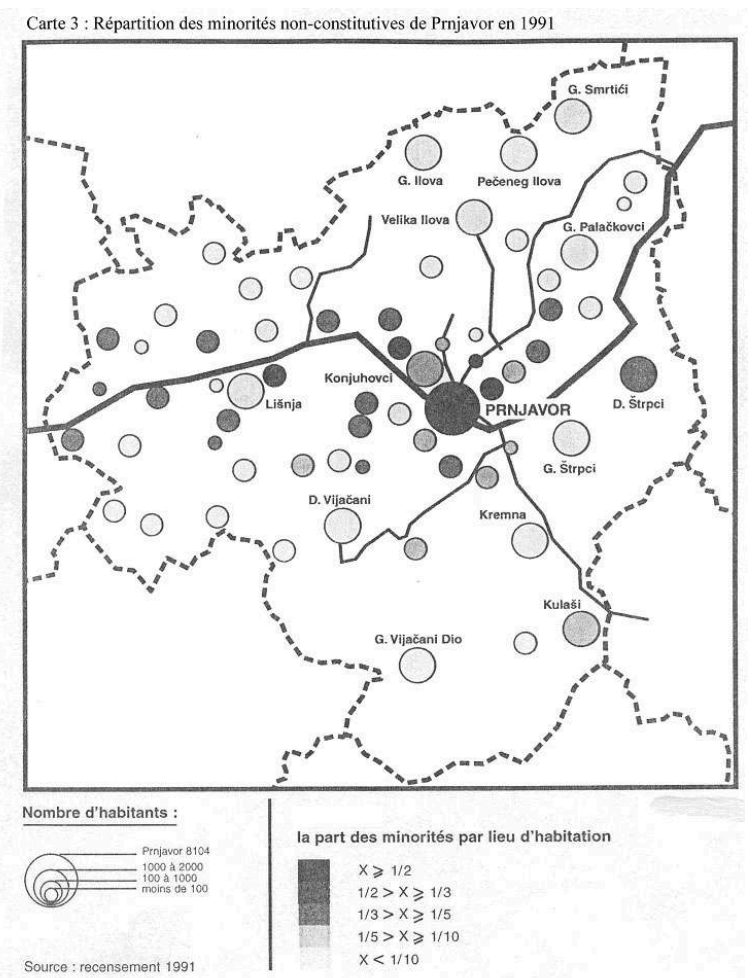

Une des principales marques de la présence de ces minorités sont les édifices religieux : églises ukrainiennes (plusieurs églises uniates et une église orthodoxe), tchèque, italienne, etc. Faute de pouvoir se regrouper dans l'espace, ces minorités ont en effet préservé leur identité nationale par le biais de paroisses en langue maternelle et d'organisations culturelles, et ce depuis leur arrivée à Prnjavor jusqu'à nos jours. Comme l'écrit B. Michel, «chanter en chœur avec ses amis des chansons issues du folklore national [est] un acte beaucoup plus chargé de signification que d'aller voter $»^{12}$. Ce caractère limité et non-territorial de revendications identitaires explique la tolérance qu'ont manifestée envers les colons les différents régimes politiques qui se sont succédés en Bosnie-Herzégovine, sauf pendant les deux dernières guerres.

\section{b) La mixité : un trait urbain}

La ville est, en Bosnie-Herzégovine, le lieu de mixité ethnique par excellence. Comme la plupart de villes fondées à l'époque ottomane, Prnjavor se situe au carrefour de plusieurs axes de communication ${ }^{13}$. Avec le repli de l'Empire ottoman sur la Save en 1699 (paix de Karlowitz) apparaît le besoin d'une voie parallèle à la rivière pour relier les parties septentrionales de l'Empire. Les récits du XIXe siècle affirment que la ville 
est fondée près d'une auberge (han), à mi-chemin entre Tešanj et Banja Luka, sur la route des caravanes commerciales. Elle apparaît pour la première fois sur une carte élaborée en 1783 par I. von Makovich, capitaine des garde-frontières en Slavonie, tandis que les sources bosniaques ne la mentionnent qu'en 1843, dans une liste des paroisses orthodoxes de la région de Banja Luka. Contrairement à ce qu'affirme Z. Nedović ${ }^{14}$, il s'agit dès l'origine d'une bourgade binationale : la première mosquée date de 1800 , et la première église orthodoxe de 1829 . On peut toutefois, avec Z. Nedović, déduire de l'étymologie du mot "prnjavor" une prépondérance originelle des chrétiens orthodoxes à Prnjavor. Cela reste une hypothèse qui n'entre pas en contradiction avec la possibilité que le monastère abandonné (qui n'a pas été localisé, faute de fouilles archéologiques) ait été transformé en auberge par des musulmans ayant quitté la Slavonie après la paix de Karlowitz.

Quoi qu'il en soit, la ville de Prnjavor a gardé jusqu'à nos jours une structuration communautaire par mahala (rue ou quartier urbain), typique des villes ottomanes.

Elles sont caractérisées par un quartier commerçant appelé čaršija, entouré de mahalas (parties résidentielles de la ville). [...] Le mahala, en tant qu'unité résidentielle de base, était formé d'une cinquantaine de maisons regroupées autour d'une mosquée [...] et d'un mekteb (école [religieuse] élémentaire). [Il] comprenait également un puits, une boulangerie, une épicerie et un cimetière. ${ }^{15}$

Carte 4 : Les mahala et les quartiers de Prnjavor dans les années 1990

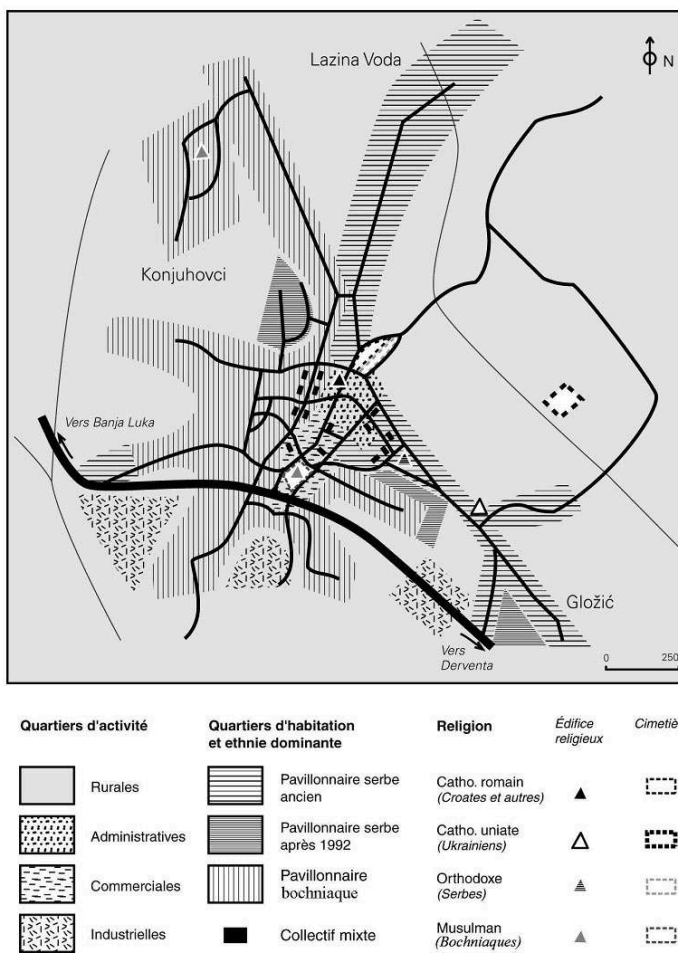

Jusqu'à la guerre de 1992-1995, on peut retrouver cette trame des mahalas à Prnjavor. L'espace urbain de cette ville d'environ 7000 habitants se divise entre son centre (čaršija) et ses périphéries pavillonnaires. D'un côté, le čaršija caractérisée avant 1992 par une forte mixité, du fait de la superposition des activités administratives et commerciales, de l'autre, les périphéries pavillonnaires à dominante bochniaque (sud et ouest) et serbe (nord et est) correspondent au noyau historique binaire de la ville. Cette répartition spatiale n'a pas été fondamentalement modifiée par l'urbanisation récente. Simplement, au lieu de préserver la vieille architecture ottomane du čaršija, le 
pouvoir communiste a préféré la remplacer par de grands ensembles de peuplement mixte (car les appartements y étaient -du moins en théorie- distribués selon les critères sociaux et non communautaires).

Ainsi, en fonction de son lieu de résidence, on pouvait avec une forte probabilité deviner à quelle communauté appartenait telle ou telle personne. L'appropriation de l'espace de la commune de Prnjavor reposait donc sur une identification communautaire par hameau, village ou quartier urbain. L'entreprise de séparation territoriale des communautés pendant la guerre a su utiliser cette donne.

\section{La guerre de 1992-1995}

Les événements qui ont marqué les années de guerre à Prnjavor sont tombés doucement dans l'oubli, à force de questions restées sans réponse. Les habitants interrogés en 1999 étaient plutôt préoccupés par un quotidien très difficile, si bien que, lorsqu'on les amenait à parler de cette période, ils avaient l'air de déterrer un mauvais souvenir enseveli sous beaucoup d'autres. C'est comme si, au fond, ils étaient contents de ne plus avoir vraiment à s'en soucier. Mais ils étaient conscients de faire partie des gens les moins affectés par la guerre.

\section{a) La violence physique et symbolique}

Les premières élections démocratiques en Bosnie-Herzégovine, le 18 novembre 1990, furent marquées à Prnjavor par la victoire du principal parti nationaliste serbe, le Parti démocratique serbe (Srpska Demokratska Stranka-SDS). Les préparatifs pour la guerre commencèrent dès le printemps 1991, avec la mobilisation générale des réservistes de l'Armée populaire yougoslave (Jugoslovenska Narodna Armija - JNA), impliquée dans les opérations militaires en Croatie. Comme beaucoup d'autres communes bosniaques, Prnjavor eut aussi sa propre milice locale: les "Loups de Vučijak". D'après le compterendu du commandant de la brigade de Prnjavor, au mois de juin 1991, 23 volontaires partirent combattre l'armée croate dans la région de la Krajina (Croatie), sous le commandement d'un petit brigand, Veljko Milanković. Il en revinrent enrichis par le pillage des maisons abandonnées (serbes et croates), pour repartir ensuite en Slavonie orientale. Ils restèrent actifs en Croatie jusqu'au début de la guerre en BosnieHerzégovine (mars-avril 1992).

Lorsque la JNA fit appel aux réservistes de Prnjavor, 140 d'entre eux (et pas seulement des Bochniaques et des Croates) refusèrent d'aller combattre en Croatie : ils furent renvoyés chez eux et la majeure partie d'entre eux furent immédiatement licenciés. Les autres restèrent engagés en Croatie jusqu'à l'arrivée de la Force de protection des Nations-Unies (Forpronu) en juin 1992. Après leur retour, les soldats de Prnjavor participèrent à la prise de contrôle du "corridor de Brčko", au nord de la Bosnie, qui permettait le passage de l'approvisionnement en provenance de la Serbie. Jusqu'à la fin de la guerre en 1995, ils participèrent aussi aux combats dans la région de la Bosanska Krajina, et à la défense de la ligne de front en Bosnie centrale.

Il faut préciser que toutes ces activités militaires étaient organisées dans le cadre normal des lois yougoslaves sur la mobilisation, comme si c'était encore la période titiste. Aussi, tous les citoyens sans distinction d'appartenance nationale étaient appelés à combattre, au nom de la "défense" de la région. Les non-Serbes devaient donc 
choisir entre la collaboration et des sanctions juridiques. L'économie de la commune était à dominante agraire, mais tout le secteur industriel était étatisé, d'où la possibilité pour le SDS de licencier les récalcitrants.

Il en va de même pour le volet médiatique des préparatifs du nettoyage ethnique. La radio locale, qui avait couvert la manifestation pacifiste du 5 mars 1992, dernière tentative d'arrêter la guerre qui s'annonçait, fut immédiatement saisie, et son directeur remplacé par un homme du SDS. Elle se mit alors à diffuser les communiqués officiels, des reportages en provenance du front, et divers chants nationalistes serbes. En juin 1992, cette radio accusa les Bochniaques du village de Lišnja de posséder des armes, ce qui servit à justifier l'attaque de l'armée contre ce village. Les termes utilisés faisaient référence à la Seconde Guerre mondiale : l'armée croate était qualifiée d'“oustachis", celle des Bochniaques de balije ${ }^{16}$.

Le terrain avait naturellement été préparé par les émissions de la Radio-Télévision de Belgrade, qui rappelait aux Serbes le danger des pogroms commis par les Oustachis, et celui du djihad des fondamentalistes. Pour avoir vu pendant les neuf mois précédant la guerre [en Bosnie-Herzégovine] des reportages authentiques montrant des cadavres et des villages brûlés en Croatie, le paysan et le citoyen serbe moyen était facilement persuadé que ces menaces étaient réelles. Il ne manquait plus que quelques détails aux couleurs locales pour compléter le tableau ${ }^{17}$.

L'épisode de Lišnja eut exactement cette fonction-là : permettre le passage de la peur au nettoyage ethnique, la psychose entretenue par les médias justifiant les exactions à l'encontre des minorités. Cependant, derrière le terme de "nettoyage ethnique" se cache toute une panoplie d'activités qui n'ont en commun que leur objectif final: l'homogénéisation du territoire sur des critères ethniques. Si les procédés classiques, décrits par N. Malcolm et bien d'autres, n'ont pas été utilisés à Prnjavor même, c'est que les configurations politiques locales les rendaient superflus. Une autre méthode fut utilisée pour s'attaquer aux minorités isolées au milieu d'un territoire dominé par les forces serbes: accuser la population minoritaire de détention illégale d'armes, puis lancer un ultimatum pour qu'elle les rende, en ouvrant ainsi la voie à une attaque militaire. Résultat : une cinquantaine de notables du village bochniaque furent arrêtés et emprisonnés dans une usine située en face du lycée.

Il faut noter qu'une résistance militaire des minorités n'avait pas de sens : elles étaient trop peu nombreuses, sans armes, éloignées d'une centaine de kilomètres des premiers territoire sous contrôle bochniaque ou croate. De nombreux Bochniaques et Croates de Prnajvor choisirent donc de s'assurer la protection du pouvoir serbe en échange de leur participation à l'effort de guerre, en combattant, en creusant des tranchées ou en effectuant d'autres travaux. Ceux qui refusaient ne pouvaient s'estimer en sécurité. Quelques meurtres commis contre les récalcitrants suffirent à convaincre les autres. Des assassinats de ce genre ont été enregistrés en 1992 dans le village bochniaque de Lišnja, et dans celui de Kulaši, à majorité croate. En juillet 1992, l'assassinat par un membre de la milice locale d'un Bochniaque qui s'était joint aux forces serbes et rentrait en permission à Prnjavor avec tout son régiment, montre que même ceux qui avaient consenti à collaborer n'étaient pas à l'abri. L'assassin fut arrêté, puis relâché quelques jours plus tard sous la pression des "Loups".

31 La violence contre les minorités à Prnjavor resta limitée à ce genre d'incidents isolés, provoqués par les milices extrémistes qui agissaient en toute impunité. Mais, même 
limitée, la violence a toujours une fonction symbolique : il suffit qu'une personne en soit victime pour que toute sa communauté se sente menacée.

Dans ce contexte, une attention particulière doit être portée à la forme la plus fréquente de violence symbolique, celle visant les édifices religieux. Les destructions de lieux de culte ne sont pas de simples actes de vandalisme: à travers les églises, les mosquées et les cimetières, ce sont bien les populations elles-mêmes qui sont visées. On peut dire que ces destructions avaient pour fonction d'annoncer des mesures plus sévères dans le cas où ces populations minoritaires refuseraient de partir. C'est pourquoi, au cours de la seule année 1992, ce sont 101 églises et 800 mosquées environ qui subirent ce genre de violence en Bosnie Herzégovine ${ }^{18}$, dont 200 édifices religieux musulmans sur 460 dans la région de Bosanska Krajina.

Aujourd'hui, il ne reste plus aucune mosquée à Prnjavor. La mosquée située au centre de la ville a été détruite par une charge explosive le 2 juin 1992. Seul reste le cimetière musulman qui l'entourait. La série de destructions de mosquées -restée inexpliquée aux dires des autorités locales, pratiquement inchangées dix ans après les événementsa commencé par l'opération des "Loups" contre le village de Lišnja. Le 2 août 1992, presque simultanément, furent endommagées l'église croate de Kulaši et l'église ukrainienne de Prnjavor. Sur la liste des lieux de culte endommagés ou détruits figurent encore les églises croates de Kulaši (27 septembre 1992), de Doline (14 mars 1993), de Drenova (15 août 1993), ainsi que toutes les mosquées, la dernière à avoir été détruite étant celle de Konjuhovci, le 30 juin 1995, jour du kurban bajram (fête du sacrifice) $)^{19}$.

\section{b) Les particularités du nettoyage ethnique à Prnjavor}

Les pouvoirs locaux de Prnjavor se défendent d'avoir été impliqués dans les destructions de lieux de culte appartenant aux populations minoritaires. Lorsque, après la destruction de leur église, le maire alla rassurer les Ukrainiens de la commune, il leur déclara qu'il s'agissait d'une erreur, et que c'était probablement l'église croate du centre ville qui était visée. Selon lui, ces actes auraient été commis par des "éléments extérieurs". Les pouvoirs locaux condamnèrent la violence en termes généraux et vagues, promirent une enquête, et on en resta là. Toutefois, le curé croate dit avoir bénéficié de la protection de la police locale contre les provocations d'éléments extrémistes.

Le sort qu'ont connu pendant la guerre les minorités non-constitutives, peu nombreuses et de mentalité loyaliste (leurs seules revendications sont d'ordre culturel et religieux), est bien meilleur que celui des Bochniaques ou des Croates. Nous étudierons ici les trois principales : les Ukrainiens, les Tchèques et les Italiens.

Ces derniers sont encore 750 dans leur village de Štivor, comme en 1992, du moins formellement. En réalité, la majorité n'y réside plus de façon permanente, et il ne reste que des personnes âgées ${ }^{20}$. En 1992, les pouvoirs locaux avaient promis aux Italiens qu'ils n'auraient pas à faire la guerre; en revanche, ils les ont laissés partir en Italie comme "travailleurs temporairement expatriés", contre le paiement d'un impôt de guerre s'élevant à $200 \mathrm{DM}$ par mois et par personne. Les Italiens de Štivor avaient été longtemps oubliés par leur pays d'origine, mais les soldats italiens de la Forpronu sont intervenus auprès des autorités locales pour que leurs lointains compatriotes reçoivent 
des passeports, et auprès du gouvernement italien pour qu'ils obtiennent des permis de séjour et de travail. Aussi, tout le monde y a trouvé son compte.

Selon les données fournies par le président de l'association culturelle tchèque locale Česka beseda ("La langue tchèque"), sur la base d'un recensement informel effectué en 1998, il y aurait dans toute la commune de Prnjavor 99 Tchèques, soit l'équivalent de la seule population de leur village de Maćino Brdo avant guerre (108 personnes en 199121). Une douzaine de familles a quitté la Bosnie, grâce à Caritas et à la Croix-Rouge, pour trouver refuge auprès de la petite communauté tchèque de Croatie, près de Daruvar. Ceux-là ont dû, comme les Bochniaques, renoncer à leurs biens, tandis que les autres ont servi loyalement dans les rangs de l'armée serbe. Lorsqu'en 1998, cette petite communauté a reçu la visite de l'ambassadeur tchèque, qui leur a proposé d'être rapatriés en République tchèque, les villageois ont décliné l'offre, manifestant ainsi un profond attachement pour leur lieu de résidence et leur vie de paysans indépendants. L'ambassadeur leur a toutefois promis de leur délivrer des passeports tchèques pour qu'ils puissent voyager.

Bien qu'étant la seule à avoir vu son église détruite, la communauté ukrainienne a aussi bien résisté que les autres. Aux dires de leur prêtre, seule source d'information en l'absence de toute statistique, une douzaine de familles au total a quitté la commune. Huit familles paysannes ont profité de liens familiaux pour émigrer au Canada. Quelques Ukrainiens urbains, artisans et universitaires, ont aussi émigré vers l'Occident, et non vers l'Ukraine. Selon le prêtre toujours, la majorité de ses paroissiens ont été retenus par l'impossibilité de quitter un territoire en plein conflit, mais aussi par leur caractère rural et leur âge avancé. Leur attachement à Prnjavor s'explique donc par une combinaison de facteurs socioculturels anciens et de circonstances immédiates. L'attitude tolérante des pouvoirs locaux à leur égard s'explique par leur insignifiance démographique et électorale. La municipalité a même participé financièrement, aux côtés des Ukrainiens locaux et de la diaspora, à la reconstruction de l'église de Prnjavor, inaugurée le 19 août $1999^{22}$.

Les interprétations historicistes du nettoyage ethnique, sur place ou à l'étranger, peuvent être résumées par les propos d'un "expert local" affirmant que «rien ne remplace chez les Serbes, dans la célébration guerrière de la victoire, la destruction des édifices religieux $»^{23}$. On voit dans le cas de Prnjavor qu'il n'y a pas la moindre trace d'une quelconque foule serbe détruisant les édifices religieux, mais des actes terroristes, commis de nuit par des professionnels du maniement des explosifs. Il peut s'agir de membres des unités spéciales, ou de miliciens. Mais derrière ces actes, il y a une volonté politique: détruire les liens d'une partie de la société avec le territoire qu'elle habite, frapper des figures de proue et des symboles communautaires, tout ceci afin d'inciter au départ. Cela suppose des organisateurs qu'il est difficile de nommer avec certitude. Les pouvoirs locaux semblent avoir joué la carte de la légalité transformée en appareil discriminatoire, alors que les milices semaient la terreur pour décourager ceux qui se résignaient à subir cette discrimination. Par ailleurs, les "ennemis" sont hiérarchisés en fonction de leur importance numérique : moins ils sont nombreux, plus les pouvoirs locaux se montrent modérés, voire protecteurs; plus ils sont nombreux, plus les pouvoirs locaux laissent le champ libre à des actions individuelles et aux milices locales. massive et/ou camps de concentration). La milice locale a même protégé la commune 
contre l'intrusion de milices concurrentes venues de la Serbie. Les seuls à avoir tenté une telle intrusion sont les "Aigles Blancs" de Mirko Jović, qui se sont attaqués aux riches maisons bochniaques de Prnjavor en juin 1992. Les "Loups" les ont roués de coups et traînés dans les rues de la ville, avec un écriteau au cou : «Je suis la honte du peuple serbe ». Difficile d'interpréter cet épisode de la guerre : esprit de clocher ou chasse gardée ? Quoi qu'il en soit, l'exode des Bochniaques a continué en empruntant des canaux para-légaux : pour partir, en empruntant le corridor serbe, il fallait acquérir de "vrais-faux" papiers, délivrés au prix fort par la mairie. Ces départs s'accompagnaient de la vente forcée, à vil prix, des biens bochniaques. Ce sont les pouvoirs locaux qui en ont tiré profit en renforçant leurs réseaux clientélistes.

La relative "modération" du nettoyage ethnique à Prnjavor peut s'expliquer par plusieurs facteurs. Tout d'abord, la commune n'était revendiquée que par les Serbes : ils y représentaient la majorité absolue de la population et détenaient le pouvoir local. Face à eux, la population bochniaque (15\% de la population en 1991) était regroupée à Prnjavor même et dans quelques villages, et éloignée d'une centaine de kilomètres du premier territoire majoritairement bochniaque ou des lignes de front. Cela avait un effet dissuasif contre toute tentative d'action armée. Par conséquent, les SDS n'a pas eu à craindre pour son pouvoir, et donc à recourir aux moyens les plus extrêmes. C'est aussi le cas de deux communes voisines qui ont un profil semblable (Čelinac et Laktaši). Tout semble se passer comme s'il existait un "seuil" au-dessous duquel la présence des minorités ne représentait pas une menace pour le pouvoir, exercé au nom de la communauté majoritaire. On peut également supposer que les ordres venus du sommet du pouvoir serbe n'étaient pas très précis, et qu'ils laissaient une certaine marge de manoeuvre aux décideurs locaux quant aux moyens à employer pour réaliser l'homogénéité ethnique du territoire qu'ils contrôlaient.

Si l'on observe les pires exactions perpétrées contre des civils en Bosnie-Herzégovine, on se rend compte qu'elles ont eu lieu dans des communes au profil situé aux antipodes du celui de Prnjavor. A Prijedor, par exemple, les Bochniaques représentaient une majorité relative de la population locale, et le Parti de l'action démocratique (Stranka Demokratske Akcije - SDA) y avait emporté les élections, alors que cette commune se trouve au coeur de la Bosanska Krajina serbe. Aussi, l'action militaire serbe y a-t-elle été particulièrement violente, car destinée à faire partir les civils bochniaques ${ }^{24}$. De manière plus générale, les violences ont été poussées à l'extrême à proximité des fronts d'importance stratégique comme le long du "corridor de Brčko" ou dans la vallée de la Drina où, pour assurer une continuité territoriale avec la Serbie, le SDS devait expulser les Bochniaques de Srebrenica, de Žepa ou de Višegrad. Par contre, il n'était pas politiquement vital pour les pouvoirs locaux de Prnjavor de chasser les Bochniaques de la commune. S'opposer à la milice locale qui le voulait restait toutefois un risque considérable.

\section{Un retour difficile}

L'après-guerre est marquée à Prnjavor par la présence de nombreux déplacés serbes. Ils seraient environ 4000 en 2000. Il s'agit surtout de personnes ayant quitté en 1995 les communes perdues par la Republika Srpska : Bosansko Grahovo, Glamoč et Drvar. C'est dans ces même communes, ainsi qu'à Martin Brod (Bihać), qu'ont trouvé refuge les Bochniaques de Prnjavor. Elles sont situées dans un des cantons croates de la Fédération de Bosnie-Herzégovine. Aussi, le retour des déplacés dépend d'une étrange 
valse : tant que les autorités croates de Bosnie-Herzégovine ne permettent pas le retour des Serbes dans les régions qu'elles contrôlent, il n'y aura pas de restitution des maisons bochniaques à Prnjavor, et vice versa.

Or, de part et d'autre, les autorités locales font preuve de mauvaise volonté. Ainsi, à Drvar, sur 2912 demandes de restitution de biens immobiliers (propriétés privées ou logements sociaux), seules 429 ont été traitées au cours de l'année 2000, et seulement 161 décisions favorables ont été rendues. De telles proportions, très inférieures aux moyennes de la Fédération, se retrouvent à Glamoč. Seul Bosansko Grahovo, confronté à une soixantaine de demandes, en a résolu $42 \%$ de manière favorable. Les autorités locales de Prnjavor ne font pas franchement mieux : sur environ 2000 demandes de restitution de la propriété déposées, un quart seulement a reçu une réponse, et $15 \%$ une réponse positive, soit dans 323 cas $^{25}$.

En plus de cette obstruction administrative des tribunaux, les pouvoirs locaux ont fermé en 2000 le cimetière musulman situé en centre-ville, à côté de la mosquée détruite ${ }^{26}$. Plusieurs attaques à la grenade contre des rentrants ont également été enregistrées au printemps 2000. Le quotidien Oslobodjenje du 26 mars 2000 affirme qu'il s'agit là d'une campagne organisée à la veille des élections locales, et destinée à stopper la timide vague de retours. Mais cela n'est peut-être pas la seule explication, ni la plus pertinente. Selon le représentant de la société caritative Merhamet à Prnjavor, dans de nombreux cas, les occupants de logements bochniaques, et notamment de logements sociaux, ne sont pas des déplacés mais des locaux qui, grâce à leurs relations, bénéficient ainsi d'un appartement confortable à côté de leur propre maison ${ }^{27}$. C'est ce genre de réseaux clientélistes qui s'opposent au retour. Quant aux déplacés, du fait de leur statut extrêmement précaire, ils sont facilement mobilisables par les partis extrémistes. Ce sont eux qui exigent des pouvoirs locaux une priorité à l'embauche ou un droit de préemption sur les envois humanitaires. Tout cela en fait des acteurs politiques très actifs, pouvant déstabiliser les oligarchies locales au pouvoir.

En fait, les retours n'ont commencé que très récemment. Selon le Haut commissariat aux réfugiés, il n'y avait en mars 2000 que 256 retours minoritaires enregistrés à Prnjavor depuis la fin de la guerre. Le phénomène s'est ensuite accéléré, avec 35 retours (31 Bochniaques et 4 Croates) pour le seul mois de janvier 200128. Mais le départ des Bochniaques est considéré par les politiciens locaux comme un fait accompli. Le danger pour eux ne vient pas du retour de quelques milliers de Bochniaques en soi, mais du discrédit que cela susciterait au sein de leur électorat, qui se tournerait alors vers un autre parti serbe plus radical. Encourager le retour au statu quo ante signifierait pour eux prendre le risque de perdre le pouvoir local, mais ne remettrait pas en cause le fait majoritaire serbe.

\section{NOTES}

1. Le système yougoslave établissait une différence entre les nations constitutives (narodi : Musulmans / Bochniaques, Serbes et Croates en Bosnie-Herzégovine) et les minorités nationales 
non-constitutives (narodnosti). Les autres minorités nationales présentes à Prnjavor étaient les Albanais, les Allemands, les Hongrois, les Macédoniens, les Monténégrins, les Polonais, les Roms, les Russes, les Ruthènes, les Slovaques et les Slovènes.

2. L'IPTF a probablement sous-estimé le nombre de Croates (275), car le prêtre catholique de Prnjavor nous a affirmé en mars 1999 avoir environ 500 paroissiens. Elle a par ailleurs surestimé le nombre des "Autres", qui a difficilement pu passer de 3000 environ à 7000 entre 1991 et 1999. La proportion de Serbes reste cependant assez crédible.

3. Mala Enciklopedija Prosveta, Belgrade : Prosveta, 1969, t. I, p. 374.

4. Tarlać (G.), « Grad koji se ne pamti » (La ville dont on ne se souvient pas), Reporter (Banja Luka), (79), 1999.

5. Sources : recensements austro-hongrois et yougoslaves, dans Marić (F.), Pregled pućanstva Bosne i Hercegovine izmedju 1875. i 1995. godine (Aperçu de la population de la Bosnie-Herzégovine entre 1875 et 1995), Zagreb: Katehetski Salezijanski Centar, 1996 et Kočović (B.), Etnički $i$ demografski razvoj u Jugoslaviji od 1921. do 1991. godine (Le développement ethnique et démographique en Yougoslavie de 1921 à 1991), 2 vol., Paris : Bibliothèque Dialogue, 1998.

6. Liski (B.), « Stoljeće života u novoj domovini » (Un siècle de vie dans la nouvelle patrie), Nova Dumka (Vukovar), (81), pp. 9-10 et (82), pp. 15-17, 1990.

7. Nedović (Z.), Prnjavor i njegova okolina (Prnjavor et ses environs), Doboj : Grafičar, 1999.

8. Malcolm (Noel), Bosnia, A Short History, Londres : Macmillan, 1996, pp. 142-143.

9. Selon V. Grotsky, ils se rendaient indifféremment à l'église catholique croate ou à l'église orthodoxe serbe. Voir Grotsky (Y.), Polojénia Oukraïntsiv ou Bosniï (L'installation des Ukrainiens en Bosnie), L'viv : s.e., 1909 (Repr., s.l.n.d.).

10. Communauté familiale élargie caractéristique des Slaves du Sud, qui regroupe plusieurs générations autour de l'homme le plus âgé (domaćin), et en commun les biens et les revenus du travail.

11. Voir Erić (M.), Seljaštvo i poljoprivreda Bosne i Hercegovine po austro-ugarskom okupacijom 1878-1918 (La paysannerie et l'agriculture en Bosnie-Herzégovine pendant l'occupation austrohongroise 1878-1918), Sarajevo : Seljačka knjiga, 1953, pp. 14-15.

12. Michel (B.), Nations et nationalismes en Europe Centrale, XIXème et XXème siècle, Paris : Aubier, p. 107.

13. Pour une comparaison avec d'autres "villes nouvelles" de l'époque ottomane, voir Prevelakis (G.), Les Balkans. Cultures et géopolitique, Paris : Nathan, p. 79.

14. Nedović (Z.), op. cit., pp. 25-29.

15. Smlatić (S.), Banja Luka, grad i njegove funkcije (Banja Luka : la ville et ses fonctions), Sarajevo : Svjetlost, 1978, p. 35.

16. Le terme "balije" est un terme péjoratif employé pour désigner les Bochniaques.

17. Malcolm (N.), op. cit., p. 273.

18. Gabrić (M.) et al., Croatia-Bosnia-Herzegovina, Sacral Institutions on Target, Zagreb : Hrvatski Informativni Centar, 1993, p. 90-92; et Omerdić (M.), « Porušeni i oštećeni vjerski objekti Islamske Zajednice na tlu Bosne i Hercegovine u toku agresije 1992. Godine » (Les monuments religieux de la Communauté islamique détruits et endommagés sur le sol de la BosnieHerzégovine pendant l'agression en 1992), Preporod (Sarajevo), 15 octobre 1992 (supplément spécial de huit pages).

19. Šošić (S.), Banjalučkom Timoteju (Au Timothée de Banja Luka), Zagreb : Teovizija, 1997, pp. 49-56.

20. Tarlać (G.), op. cit.

21. Gelo (J.) et al., Stanovništvo Bosne i Hercegovine : narodnosni sastav po naseljima (La population de la Bosnie-Herzégovine : la composition nationales par lieux habités), Zagreb : Državni zavod za statistiku, 1995. 
22. Holowinsky (Y.), " Parishioners in Prnjavor Celebrate the Reopening of Their Church », The Ukrainian Weekly, (40), 17 octobre 1999.

23. Marković (I.), «Srpsko-pravoslavno zaledje razaranja sakralnih objekata u Bosni i Hercegovini kao sredstvo etničkog čišćenja » (Les origines serbo-orthodoxes de la destruction des édifices religieux en tant que moyen de nettoyage ethnique), Bosna franciscana, (1), 1993, p. 101.

24. Sur le nettoyage ethnique à Prijedor, voir en particulier le « rapport Bassiouni » publié par l'ONU en juin 1994 (Rapport final de la commission d'experts constituée conformément à la Résolution 780 du Conseil de sécurité, document S/1994/674).

25. Commision for Real Property Claims, Statistics Implementation of the Property Laws, Sarajevo : CRPC, 2000.

26. U.S. Department of State, Annual Report on International Religious Freedom - Bosnia and Herzegovina, Washington, 2000.

27. Oslobodjenje, 14 septembre 1999.

28. United Nations High Commissioner for Refugees, Registered Minority Returns 01/01/2001 to 31/01/2001 in Bosnia and Herzegovina, Sarajevo, 2001.

\section{INDEX}

Index géographique : Bosnie-Herzégovine, Prnjavor

Mots-clés : Minorités nationales, Nettoyage ethnique

\section{AUTEUR}

\section{NIKOLA GULJEVATEJ}

Etudiant en troisième cycle à l'Institut d'études politiques de Paris. L'article s'appuie largement sur un mémoire de maîtrise de géographie soutenu à la Sorbonne en 1999. 